\title{
A Novel Iterative Algorithm for Recovering Shape and 3D Information using Photometric Stereo with Point Light Sources in Attenuating and Scattering Media
}

\author{
K. Nagesh \\ Adjunct Professor, \\ CUTM, Parlakhemundi, \\ Gajapati district, Odisha, India
}

\author{
D. Nageswara Rao \\ Vice Chancellor, \\ CUTM, Parlakhemundi, \\ Gajapati district, Odisha, India
}

\author{
Song K. Choi \\ Professor, \\ University of Hawaii, \\ Honolulu, HI, USA
}

\begin{abstract}
When photometric stereo technique is used to recover shape and 3D information of an object from multiple images, it is common to assume that the light sources being used are collimated. When the light sources that are being used are actually point light sources, as in robot applications for weld seam inspection or underwater imaging, such an assumption causes significant error. The error increases further when the imaging system is deployed in an attenuating and scattering media. In such situations, a purely analytical solution is not possible. Current work proposes a novel iterative algorithm for recovering shape and 3D information in such situations.
\end{abstract}

\section{General Terms}

Underwater Robotic Vehicles, Industrial Robots, Computer Vision, Algorithm.

\section{Keywords}

Photometric Stereo, Point Light Sources, Attenuating Media, Scattering Media.

\section{INTRODUCTION}

Photometric stereo technique [1] is a popular technique used to recover shape and 3D information of an object from its multiple images, each taken with same imaging system at same position but with a different light source at a different position. While using this technique, it is common to assume that the light sources being used are collimated.

However, very often, the light sources being used in practice, such as in weld seam inspection and underwater imaging applications, are actually point light sources. When these light sources are approximated as collimated light sources, it causes significant error in the recovered shape and 3D information of the object. The error increases further when the imaging process is undertaken in an attenuating and scattering media.

In such complex situations, a pure analytical solution is not possible. Current work proposes a novel iterative algorithm for recovering $3 \mathrm{D}$ information in such situations.

\section{PROBLEM}

Consider a coordinate system as shown in Figure 1 in which the origin is located at the camera image plane and the z-axis points along the optical axis toward the object.

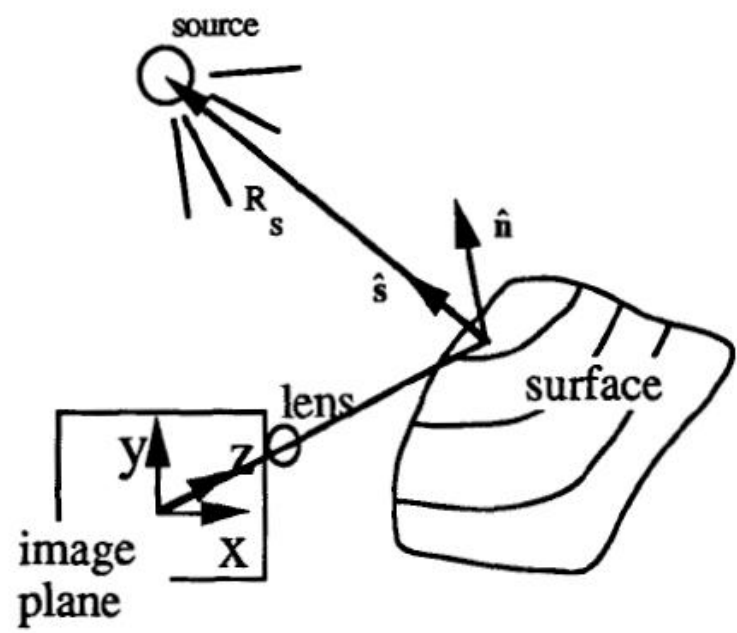

Figure 1: Coordinate system

Using radiometric terminology suggested by the U.S. National Bureau of Standards [2], we define the irradiance (E) as the incident radiant flux per unit area of the receiving surface. The radiant flux density $(\mathrm{P})$ is the radiant power per unit area normal to the ray.

The irradiance $(\mathrm{E})$ of a surface illuminated by a light source is related to the radiant flux density $(\mathrm{P})$ at the surface by Equation 1.

$$
\mathrm{E}=\mathrm{P}(\mathbf{n} \cdot \mathbf{s})
$$

Here $\mathbf{n}$ is the unit outward surface normal and $\mathbf{s}$ is a unit vector pointing from the surface toward the light source.

Following the procedure suggested by [3], it is convenient to substitute for the dot product in Equation 1 in terms of the gradient angles of the reflecting surface element.

The two unit vectors $\mathbf{n}$ and $\mathbf{s}$ can be specified by Equations 2 and 3 respectively.

$$
\begin{aligned}
& \mathbf{n}=[-1, \mathrm{p}, \mathrm{q}]^{\mathrm{T}} \\
& \mathbf{s}=\left[-1, \mathrm{p}_{\mathrm{s}}, \mathrm{q}_{\mathrm{s}}\right]^{\mathrm{T}}
\end{aligned}
$$

Here $\mathrm{p}$ and $\mathrm{q}$ are the surface gradient components: $\mathrm{dz} / \mathrm{dx}$ and $\mathrm{dz} / \mathrm{dy}$, and $\mathrm{p}_{\mathrm{s}}$ and $\mathrm{q}_{\mathrm{s}}$ are the surface gradient components of a plane normal to the vector $\mathbf{s}$.

Equation 1 can be combined with Equations 2 and 3 and rewritten as Equation 4.

$$
\mathrm{E}=\mathrm{P} \frac{\left(1+\mathrm{pp}_{\mathrm{s}}+\mathrm{qq}_{\mathrm{s}}\right)}{\sqrt{\left(1+\mathrm{p}^{2}+\mathrm{q}^{2}\right)} \sqrt{\left(1+\mathrm{p}_{\mathrm{s}}^{2}+\mathrm{q}_{\mathrm{s}}^{2}\right)}}
$$


If the surface is Lambertian, the image brightness $(F)$ produced when observing the object from any direction is proportional to the irradiance (E). The constant of proportionality depends only on the reflectivity of the surface ( $\rho$ ) and the optics of the imaging system. The dependence on the optics is generally ignored since it is specific to the imaging system and is easily obtained through calibration. Hence, the image brightness $(\mathrm{F})$ is related to the irradiance $(\mathrm{E})$ by Equation 5 .

$$
\mathrm{F}=\rho \mathrm{E}
$$

Equations 5 can be combined with Equation 4 and rewritten as Equation 6.

$$
\mathrm{F}=\rho \mathrm{P} \frac{\left(1+\mathrm{pp}_{\mathrm{s}}+\mathrm{qq}_{\mathrm{s}}\right)}{\sqrt{\left(1+\mathrm{p}^{2}+\mathrm{q}^{2}\right)} \sqrt{\left(1+\mathrm{p}_{\mathrm{s}}^{2}+\mathrm{q}_{\mathrm{s}}^{2}\right)}}
$$

If the direction (s) and radiant flux density (P) of the illumination are known at each point on the surface, three independent evaluations of Equation 6 for each surface element are sufficient to solve for the two unknown surface gradient components (p, q) and the unknown surface reflectance $(\rho)$ at the surface element.

Once the surface gradient components $(p, q)$ are computed for each surface element, shape and 3D depth (z) map of the object can be computed using Equation 7 .

$$
\mathrm{z}=\mathrm{z}_{0}+\int(\mathrm{pdx}+\mathrm{q} d \mathrm{~d})
$$

Here $\mathrm{z}_{0}$ is the depth of a known point on the object i.e. 'datum depth'.

[1] accomplishes this by assuming that the light source is collimated and uniform so that $\mathrm{s}$ and $\mathrm{P}$ are spatially invariant. Three images using same imaging system but three different light sources, as shown in Figure 2, provide the required three independent evaluations of Equation 6. This technique is known as 'photometric stereo'.

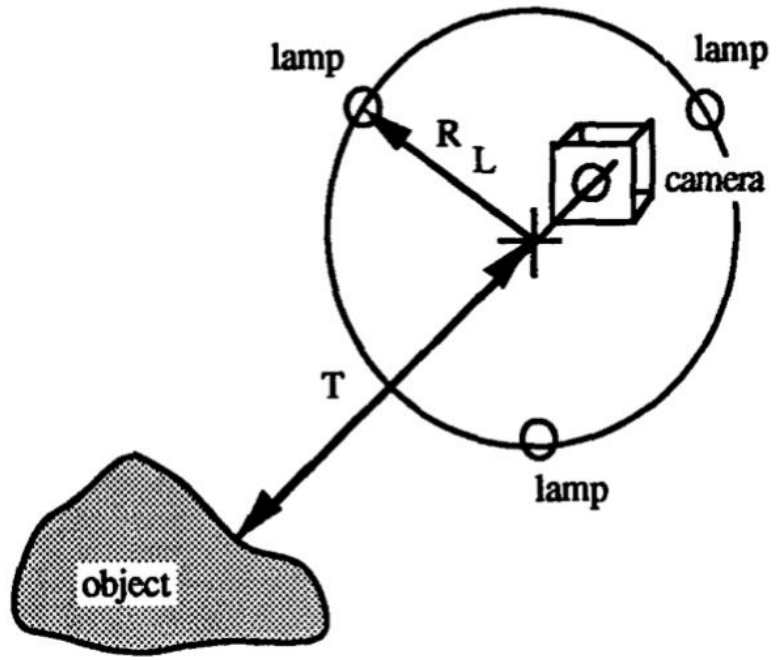

Figure 2: Light source arrangement

In practice this can be expected to work well as long as the difference in the depth between the nearest and furthest surface elements on the object being observed is small compared to the distance of the object from the light source.

In the case of a mobile robot carrying its own lamps, or cooperating robots trying to minimize backscatter into the camera [4], as in weld seam inspection and underwater applications, the light source is likely to be close to the objects being viewed. In such cases, the light sources are better approximated as point light sources. To assume they are collimated light sources causes significant error in the recovered shape and 3D information of the object. The error increases further when the imaging process is undertaken in an attenuating and scattering media.

\section{SOLUTION}

\subsection{Using Point Light Sources}

When using point light sources, the local radiant flux density (P) can be expressed in terms of the distance from the point light source (Rs) as given in Equation 8.

$$
P=\frac{I_{0}}{R_{S}^{2}}
$$

Here $I_{0}$ is the radiant intensity of an isotropic point light source.

Since distance and direction to point light source $\left(\mathrm{R}_{\mathrm{s}}\right)$ varies from one surface element to another, $\mathrm{p}_{\mathrm{s}}$ and $\mathrm{q}_{\mathrm{s}}$ themselves vary from one surface element to another. Hence, having three images is no longer sufficient to solve Equation 6 and compute $\mathrm{p}, \mathrm{q}$ and $\rho$ at each surface element.

\subsection{In Attenuating Media}

In addition, if the operation takes place in a turbid or smoky medium, attenuation will be present so that the radiant flux density $(\mathrm{P})$ will vary as a function of position for each of the surface elements on the object as described in Equation 9.

$$
\mathrm{P}=\mathrm{I}_{0} \frac{\mathrm{e}^{-\frac{\mathrm{R}_{\mathrm{s}}}{\beta}}}{\mathrm{R}_{\mathrm{s}}^{2}}
$$

Here $\beta$ is the characteristic attenuation length.

\subsection{In Scattering Media}

When the medium is also scattering, if we assume it is homogeneous and not highly dense, as in smoky welding environment or murky water, we can use single scattering model (Figure 3) [5].

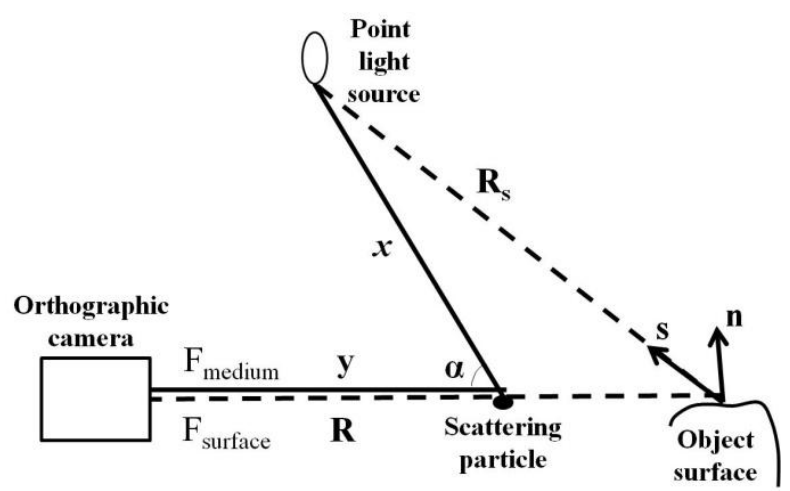

Figure 3: Single scattering model

Using single scattering model, the image brightness (F) due to a particle in the medium is given by Equation 10 .

$$
\mathrm{F}_{\text {medium }}=\left(\mathrm{P} \mathrm{e}^{-\frac{x}{\beta}}\right)(\gamma \mathrm{S}(\mathrm{g}, \alpha))\left(\mathrm{e}^{-\frac{\mathrm{y}}{\beta}}\right)
$$

Here $\gamma$ is scattering coefficient i.e. fraction of the incident flux scattered by a unit volume of the medium in all directions, $\mathrm{S}(\mathrm{g}, \alpha)$ is phase function i.e. angular scattering distribution of the incident flux, $\mathrm{x}$ is the distance of the scattering particle from the point light source and $\mathrm{y}$ is the distance of the scattering particle from the origin of the coordinate system. 
$\mathrm{S}(\mathrm{g}, \alpha)$ is assumed to be a smooth, low-order polynomial of $\cos \alpha$, where $\alpha$ is the angle between incident and scattered directions. Its first-order approximation is given by Equation 11 .

$$
\mathrm{S}(\mathrm{g}, \alpha)=\frac{1}{4 \pi}(1+\mathrm{g} \cos \alpha)
$$

Here $g$ is the forward scattering parameter that controls the shape of the phase function and $€(-1,1)$.

The total image brightness of a pixel corresponding to a surface element due to scattering by all particles is obtained by integrating Equation 10 along viewing direction to surface element from 0 to $\mathrm{R}$.

This together with irradiance due to the surface element produces the final image brightness $(\mathrm{F})$ as given by Equation 12.

$$
\mathrm{F}=\mathrm{F}_{\text {medium }} \delta(\mathrm{x}<\mathrm{R})+\mathrm{F}_{\text {surface element }} \delta(\mathrm{x}=\mathrm{R})
$$

Here $\delta$ is Dirac delta function.

\subsection{A Novel Iterative Algorithm as \\ Solution}

Since it is assumed that the position of the lamp is known with respect to the camera, the surface element to lamp vector (Rs) (which is s) may be expressed using Equation 13 in terms of the depth of the surface element from camera origin $(\mathrm{z})$ via an appropriate transformation, as shown in Figure 4.

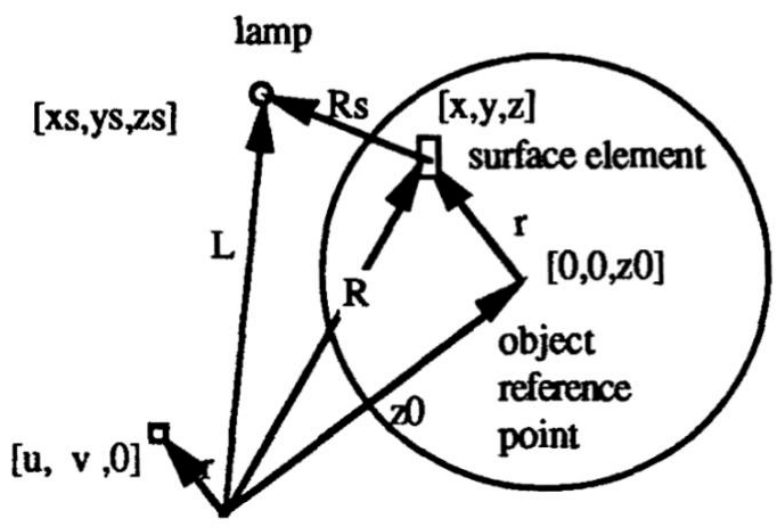

Figure 4: Determination of $\mathbf{R}_{\mathrm{s}}$

$$
\mathrm{R}_{\mathrm{S}}=\mathrm{L}-\mathrm{R}=\mathrm{L}-\left[\frac{\mathrm{uz}}{\mathrm{f}} \frac{\mathrm{vz}}{\mathrm{f}} \mathrm{z}\right]
$$

Here $\mathrm{L}$ is the known vector from the camera origin to the lamp and $\mathrm{R}$ is the vector from the camera origin to the surface element under consideration.

The components of $\mathrm{R}$ from perspective projection are $\mathrm{uz} / \mathrm{f}$, $\mathrm{vz} / \mathrm{f}$ and $\mathrm{z}$ where $\mathrm{u}$ and $\mathrm{v}$ are the image pixel coordinates in the $\mathrm{x}$ and $\mathrm{y}$ directions respectively and $\mathrm{f}$ is the focal length of the optical system.

The direction of the vector $\mathbf{R}_{\mathrm{S}}$ (which is $\mathbf{s}$ ) provides us with the quantities $\mathrm{p}_{\mathrm{s}}$ and $\mathrm{q}_{\mathrm{s}}$.

Given the camera depth (z) to each surface element, the above indicates that it is possible to solve the above set of equations for the surface gradient components even though the light sources are not uniform and collimated and the medium is attenuating and scattering.

This depth information, however, is not available for each surface element. If one had a complete depth map, there would be no need to use photometric stereo to ascertain $\mathrm{p}$ and $\mathrm{q}$, since they could be obtained directly from their definitions $(\mathrm{p}=\mathrm{dz} / \mathrm{dx}, \mathrm{q}=\mathrm{dz} / \mathrm{dy})$

Fortunately, the depth (z) of each surface element can be expressed in terms of a datum depth (z0) and the surface gradient using Equation 7

Hence, if there is sparse range data available, one datum for each object in the scene, such as can be specified by operator in the case of weld seam inspection application or obtained from sonar in the case of underwater imaging application, it should be possible to solve the above set of equations iteratively for $\mathrm{p}$ and $\mathrm{q}$.

The proposed novel iterative algorithm to calculate the shape and $3 \mathrm{D}$ information of an object using photometric stereo with point light sources in attenuating and scattering media can be summarized as follows:

(1) Assume the object to be a flat plane $(\mathrm{p}=\mathrm{q}=0)$ at a known global depth z0.

(2) Use the values of $p$ and $q$ in Equation 7 together with the global depth $(\mathrm{z} 0)$ to obtain depth $(\mathrm{z})$ of each surface element.

(3) Compute the light source direction and range from the surface from Equation 13.

(4) Compute the flux density for each surface element from Equations 4 and 9.

(5) Solve the three evaluations of the Equation 6 simultaneously for each surface element to determine the surface gradient components (p,q), after subtracting contribution from scattering particles given by Equation 10 .

(6) Return to step (2) until the desired degree of convergence is obtained.

\section{CONCLUSIONS}

The method of photometric stereo, generally used for collimated light sources, has been extended using a novel iterative algorithm to include the case of point light sources in attenuating and scattering media. The algorithm requires only a single range estimate for each of the objects in the scene.

\section{ACKNOWLEDGMENTS}

We thank Prof. Joel S. Fox and the reviewers for their insightful comments.

\section{REFERENCES}

[1] Woodham, R.J., 1980 . Photometric method for determining surface orientation from multiple images. OptEng, 19(1). .

[2] Nicodemus, F.E.. Richmond, J.C., Hsia, Ginsberg, I.W. and Limperk, T., 1977. NBS Monograph 160, National Bureau of Standards, Washington, D.C.

[3] Horn, B.K.P., 1975. Obtaining Shape from Shading Information. In: Winston, P.H. (ed.). Psychology of Computer Vision. McGraw-Hill Book Co., New York.

[4] Turner, RM., Turner, E.H., Fox, J.S., Blidberg, D.R., 1991. Multiple Autonomous Vehicle Imaging System. 7th Int. Symp. on Unmanned Untethered Submersible Technology. Sept., Durham, New Hampshire.

[5] Narasimhan, S. G., Nayar, S. K., Sun, B., Koppal, S. J., 2005. Structured light in scattering media. October. 